\title{
Condutividade Elétrica de Misturas de Poliestireno/ Polibutadieno e Poliestireno/Copolímero em Bloco de Estireno-Butadieno Contendo Negro de Fumo.
}

\author{
Bluma G. Soares, Angelo J. B. Ferreira e Sérgio Camargo J r.
}

Resumo: A condutividade elétrica de compósitos contendo negro de fumo extra condutor em sistemas envolvendo poliestireno/ polibutadieno (PS/PBD) e poliestireno/copolímero em bloco de estireno-butadieno (PS/ $\mathrm{SB}$ ) foi investigada. A mistura PS/SB apresentou menores limiares de percolação do que misturas PS/PBD. Análises através de microscopia eletrônica de varredura indicaram que, no caso das misturas PS/PBD, as partículas de negro de fumo estão aglomeradas e não formam um caminho contínuo, característico de condução elétrica. Investigações a respeito da formação de interações físicas entre polímero-negro de fumo (bound rubber) indicaram também maior adesão entre polímero-NF quando se utilizou o sistema PS/PBD.

Palavras-chave: Compósitos condutores, negro de fumo, misturas poliméricas.

\section{Introdução}

Negro de fumo (NF) tem sido bastante empregado no desenvolvimento de compósitos poliméricos condutores devido à sua capacidade de conduzir a eletricidade em uma determinada faixa. Dentre as principais aplicações destacam-se a produção de artefatos capazes de dissipar cargas de natureza eletrostática, capazes de promover blindagem eletromagnética, entre outras. ${ }^{[1]} \mathrm{O}$ processo de condução elétrica nestes compósitos é complicado e depende fundamentalmente da concentração de negro de fumo utilizada, da viscosidade do meio e da natureza da matriz polimérica. ${ }^{[2-5]}$ Quando a sua concentração é muito baixa, as partículas se encontram completamente dispersas na matriz polimérica e o material resultante apresenta características isolantes, semelhantes às da matriz. A partir de uma determinada concentração de negro de fumo, a condutividade aumenta drasticamente devido à formação de uma rede contínua de condução, composta pelas partículas condutoras do NF em contato mútuo. ${ }^{[2-7]}$ Esta concentração crítica de NF é conhecida como limiar de percolação e deve ser a mais baixa possível de forma a preservar as propriedades mecânicas do material polimérico, minimizar problemas relacionados ao processamento e diminuir o custo do compósito final. Vários artifícios têm sido empregados para se atingir estes objetivos. A maioria deles baseia-se na otimização das condições de processamento e moldagem, ${ }^{[2-5]}$ na utilização de NF com distribuição de tamanho de partícula e porosidade adequadas ${ }^{[7]} \mathrm{e}$ na utilização de uma matriz polimérica capaz de fornecer um balanço adequado entre as interações NFpolímero, polímero-polímero e NF-NF. ${ }^{[8-9]}$ Neste sentido, a utilização de sistemas poliméricos 
multifásicos tem despertado a atenção de vários pesquisadores da área. ${ }^{[4,6,10-14]}$

Para que o composto multifásico seja condutor a uma concentração de NF bem menor, é preciso que dois principais requisitos sejam preenchidos. Um deles envolve a distribuição heterogênea do NF dentro da matriz polimérica. Este comportamento é normalmente observado uma vez que o NF apresenta afinidade diferente com os vários polímeros conhecidos. ${ }^{[12]}$ A outra condição importante diz respeito à morfologia da mistura. Neste caso, duas situações podem acontecer: uma das fases poliméricas é contínua e as partículas de NF estão localizadas nesta fase contínua; ou as duas fases são co-contínuas e o NF se localiza preferencialmente na fase que estiver presente em menor quantidade ou melhor, na interface. ${ }^{[4]}$ Estando localizadas em uma das fases contínuas ou na interface, a chance de contato entre as partículas condutoras aumenta sensivelmente, favorecendo então a condução elétrica a uma concentração de NF bem menor.

A maioria dos sistemas multifásicos empregados nestes estudos consiste de misturas poliméricas contendo dois homopolímeros imiscíveis entre si. Recentemente porém, constatamos que baixos limiares de percolação podem ser alcançados em sistemas envolvendo copolímero em bloco e suas misturas com homopolímero. ${ }^{[15-16]}$ De fato, foi observado um limiar de percolação da ordem de $3.0 \%$ em peso $(1,5 \%$ em volume) de NF em compósitos constituídos de copolímero em bloco de estireno-butadieno (SB) e suas misturas com poliestireno. Os valores de limiar de percolação foram inclusive inferiores àqueles observados para a mistura imiscível constituída de poliestireno-polibutadieno.

O objetivo deste trabalho é, portanto, discutir a condutividade elétrica da mistura física de PS/PBD contendo diferentes concentrações de NF e tentar discutir as diferenças entre o comportamento destas misturas com aquelas envolvendo o copolímero em bloco. Para tanto, foi analisada também a morfologia dos dois sistemas.

\section{Parte Experimental}

\section{Materiais}

Poliestireno (PS) (EDN-89G; $\mathrm{M}_{\mathrm{n}}=58.000 ; \mathrm{M}_{\mathrm{w}}$ $=210.800$; densidade $=1,05$; índice de fluidez $\left(160^{\circ} \mathrm{C} /\right.$
$15 \mathrm{Kg})=0,55 \mathrm{~g} / 10 \mathrm{~min}$; cedido pela EDN Poliestireno do Sul), copolímero em bloco de estireno-butadieno (SB) (BASF Styrolux 656C; $M_{n}=102.100 ; M_{w}=$ 143.000; razão PS/PBD = 83:17 \% em peso; $M_{n}$ do segmento de PS $=84.200$; índice de fluidez $\left(160^{\circ} \mathrm{C} /\right.$ $15 \mathrm{Kg})=4,60 \mathrm{~g} / 10 \mathrm{~min}$ ) e negro de fumo extra condutor (NF) (DEGUSSA Printex XE-2; DBP = $370 \mathrm{ml} /$ $100 \mathrm{~g} ; \mathrm{BET}=1000 \mathrm{~m}^{2} / \mathrm{g}$ ) foram secos em estufa a vácuo durante $24 \mathrm{~h}$. Polibutadieno (PBD) (Coperbo; $\left.M_{n}=200.000 ; M_{w}=410.000\right)$ foi também utilizado nas misturas sem purificação.

\section{Preparação das Misturas}

Foram preparadas misturas de PS/SB na proporção de 40:60\% em peso, por apresentar um relativamente baixo limiar de percolação. ${ }^{[15]}$ No caso das misturas PS/PBD, o conteúdo de polibutadieno empregado foi de $10 \%$ em relação à quantidade de $\mathrm{PS}, \mathrm{o}$ que corresponde à mesma proporção de butadieno nas misturas PS/SB estudadas neste trabalho. Os polímeros foram primeiramente adicionados em misturador interno Haake a $160^{\circ} \mathrm{C}$ e $20 \mathrm{rpm}$. Após um minuto, o NF foi adicionado e a velocidade do motor foi elevada para 60rpm durante $10 \mathrm{~min}$. As misturas foram então moldadas por compressão a $200^{\circ} \mathrm{C}$ ou $250^{\circ} \mathrm{C}$ durante $10 \mathrm{~min}$, a uma pressão de $6,7 \mathrm{MPa}$, em placas de $16 \mathrm{~cm}^{2}$ e cerca de $1 \mathrm{~mm}$ de espessura.

\section{Caracterização}

A resistividade elétrica foi medida com um eletrômetro Keithley 617, usando contatos na configuração do tipo "sanduiche". A área do eletrodo era de $9 \mathrm{~cm}^{2}$. Cola à base de prata foi também utilizada entre a amostra e o eletrodo para minimizar resistência de contato. Para cada mistura foram preparadas três amostras na média. $\mathrm{O}$ tempo de aplicação do campo elétrico foi de 1 minuto, quando se observou estabilização nos valores de resistência. As medidas foram realizadas com valores fixos de corrente aplicada e que dependem da escala de resistência considerada, conforme ilustra a Tabela abaixo:

\begin{tabular}{cc|cc}
\hline Escala & Corrente & Escala & Corrente \\
\hline $2 \mathrm{~K} \Omega$ & $100 \mu \mathrm{A}$ & $200 \mathrm{M} \Omega$ & $10 \mathrm{~mA}$ \\
$20 \mathrm{~K} \Omega$ & $100 \mu \mathrm{A}$ & $2 \mathrm{G} \Omega$ & $1 \mathrm{~mA}$ \\
$200 \mathrm{~K} \Omega$ & $10 \mu \mathrm{A}$ & $20 \mathrm{G} \Omega$ & $1 \mathrm{~mA}$ \\
$2 \mathrm{M} \Omega$ & $1 \mu \mathrm{A}$ & $200 \mathrm{G} \Omega$ & $1 \mathrm{~mA}$ \\
$20 \mathrm{M} \Omega$ & $100 \mu \mathrm{A}$ & & \\
\hline
\end{tabular}


Determinações relacionadas com a formação de gel físico entre polímero e NF foram conduzidas, dissolvendo-se a amostra de massa conhecida (cerca de $0,5 \mathrm{~g}$ ) em tolueno, à temperatura ambiente. Após 48 horas em contato com o solvente, a emulsão formada foi submetida à centrifugação durante $2 \mathrm{~h}$ a $7.000 \mathrm{rpm}$. A camada orgânica, contendo o polímero além de partículas de NF ligadas ao polímero, foi então separada. Nova porção de tolueno foi, então, adicionada no tubo contendo o material não extraído e o processo foi repetido por mais três vezes. O material não extraído foi seco, pesado e submetido à análise termogravimétrica, de acordo com procedimento descrito na literatura. ${ }^{[17]}$ Para esta análise, foi utilizado um aparelho PerkinElmer TGA-7, sendo as amostras aquecidas sob nitrogênio a uma velocidade de $20^{\circ} \mathrm{C} . \mathrm{min}^{-1}$ até $750^{\circ} \mathrm{C}$.

Análises de microscopia eletrônica de varredura (MEV) foram realizadas em microscópio JEOL 5300, utilizando-se superfícies das amostras fraturadas sob nitrogênio líquido e cobertas com uma camada de ouro.

\section{Resultados e Discussão}

A condutividade elétrica de misturas envolvendo poliestireno e polibutadieno (PS/PBD) ou poliestireno e copolímero em bloco de estirenobutadieno (PS/SB) foi determinada em função da concentração de NF e da temperatura de moldagem. A Tabela 1 apresenta os resultados de resistividade elétrica, onde se observa um limiar de percolação bem mais baixo para as misturas envolvendo o copolímero em bloco.

As misturas PS/PBD apresentam características isolantes com a adição de até $6 \%$ em peso de NF, para

Tabela 1. Resistividade elétrica de misturas PS/SB e PS/PBD em função da concentração de negro de fumo e da temperatura de moldagem

\begin{tabular}{ccccc}
\hline \multirow{2}{*}{$\begin{array}{c}\text { Conteúdo } \\
\text { de NF } \\
\text { \% em peso) }\end{array}$} & \multicolumn{3}{c}{ Resistividade Elétrica, $\Omega . c m$} \\
\cline { 2 - 5 } & PS/SB $(40: 60 \%)$ & \multicolumn{2}{c}{ PS/PBD $(90: 10 \%)$} \\
\cline { 2 - 5 } 1,9 & $200{ }^{\circ} \mathrm{C}^{\mathrm{a}}$ & $250{ }^{\circ} \mathrm{C}^{\mathrm{a}}$ & $200{ }^{\circ} \mathrm{C}^{\mathrm{a}}$ & $250{ }^{\circ} \mathrm{C}^{\mathrm{a}}$ \\
\cline { 2 - 5 } 2,4 & $2 \times 10^{13}$ & $2 \times 10^{13}$ & $2 \times 10^{13}$ & $2 \times 10^{13}$ \\
2,8 & $1 \times 10^{13}$ & $5 \times 10^{13}$ & $2 \times 10^{13}$ & $2 \times 10^{13}$ \\
3,3 & $3 \times 10^{9}$ & $2 \times 10^{4}$ & $2 \times 10^{13}$ & $2 \times 10^{13}$ \\
3,6 & $6 \times 10^{6}$ & $5 \times 10^{3}$ & $2 \times 10^{13}$ & $2 \times 10^{13}$ \\
4,4 & $8 \times 10^{4}$ & $2 \times 10^{13}$ & $2 \times 10^{13}$ & $1 \times 10^{7}$ \\
6,0 & $4,5 \times 10^{4}$ & $3 \times 10^{3}$ & $2 \times 10^{13}$ & $4 \times 10^{4}$
\end{tabular}

a) temperatura usada na moldagem por compressão, durante 10 minutos amostras moldadas a $200^{\circ} \mathrm{C}$. Quando as misturas foram moldadas a $250^{\circ} \mathrm{C}$, a transição isolante - condutor foi observada a uma concentração de NF mais baixa, ou seja, a cerca de $4,4 \%$ de NF. No caso das misturas $\mathrm{PS} / \mathrm{SB}$, o início da condução elétrica ocorreu a cerca de $2,4 \%$, quando moldadas a $250^{\circ} \mathrm{C}$. Este comportamento pode ser atribuído à coalescência dos domínios de PBD com o aumento da temperatura de moldagem. Considerando que o NF possui maior afinidade pela fase elastomérica, ${ }^{[18,19]} \mathrm{o}$ processo de moldagem deve estar favorecendo a formação de fase contínua de PBD e com isto o contato entre as partículas condutoras. Mudanças morfológicas e coalescência de fases com as condições de moldagem têm sido discutidas na literatura para sistemas heterofásicos. ${ }^{[20]}$

Para avaliar o grau de continuidade em ambas as fases da mistura PS/PBD, amostras moldadas foram submetidas à extração seletiva da fase poliestireno com metil-etil-cetona (MEK). Segundo a literatura, ${ }^{[4,21]}$ o ponto de inversão de fases (morfologia co-contínua) em uma mistura heterogênea pode ser identificado através da extração seletiva de uma das fases. Quando se consegue extrair totalmente a fase em questão sem a fragmentação da amostra, ou seja, mantendo-se a estabilidade dimensional, a morfologia naquela composição da mistura é considerada co-contínua. Após a extração com MEK, a amostra PS/PBD (90:10\%) contendo $3 \%$ de $\mathrm{NF}$ e moldada a $200^{\circ} \mathrm{C}$ resultou em um pedaço não fragmentado correspondente a $8 \%$ da amostra total. Este pedaço é obviamente constituído de PBD como fase contínua, além do negro de fumo. A afirmativa de que o NF está disperso ou aderido à fase PBD é baseada em dados da literatura ${ }^{[14]}$ e na observação de que a solução de MEK contendo PS se apresentou completamente límpida, sem qualquer vestígio de NF. Uma vez que a proporção inicial de PBD e de NF era de $10 \%$ e $3 \%$, respectivamente, o pedaço relativo a $8 \%$ da amostra é composto por $1,9 \%$ de NF e $6,1 \%$ de PBD. A partir destes resultados, pode-se concluir que a mistura moldada a $200^{\circ} \mathrm{C}$ apresenta uma morfologia caracterizada por uma fase PS contínua e uma fase PBD com 63\% de continuidade.

Quando a mistura foi moldada a $250^{\circ} \mathrm{C}$, o pedaço de amostra não fragmentado obtido foi de $12 \%$, o que corresponde a 9,2\% de PBD e 2,8\% de NF. Nestas condições de moldagem, a fase PBD apresenta uma continuidade de $95 \%$, ou seja, o sistema é praticamente co-contínuo. Estas características morfológicas das misturas PS/PBD moldadas a $250^{\circ} \mathrm{C}$ podem explicar o decréscimo do limiar de percolação nestas condições de 
moldagem. Acredita-se que este mesmo fenômeno possa ocorrer se aumentarmos o tempo de moldagem.

As características morfológicas das misturas PS/SB e PS/PBD contendo 3\% de negro de fumo foram investigadas por microscopia eletrônica de varredura (MEV). A Figura 1 ilustra as micrografias das amostras criofraturadas e submetidas a polimento com o objetivo de eliminar os sinais provenientes da fratura. As misturas PS/SB moldadas a 200 e $250^{\circ} \mathrm{C}$ (Fig. 1a e 1 b, respectivamente) apresentam uma distribuição bem mais homogênea das fases. As partículas de NF são identificadas pelas regiões brancas existentes ao longo das micrografias. Embora os valores de resistividade elétrica tenham sido influenciados pela temperatura de moldagem, a morfologia da mistura não parece ter sido afetada pelo tratamento de moldagem por compressão. Pode-se sugerir que, em ambos os casos, o negro de fumo forma caminhos de condução elétrica estreitos e homogêneos além de uma provável localização do negro de fumo na interface. Segundo alguns autores, variações significativas nos valores de condutividade com a temperatura ou tempo de moldagem constituem uma forte indicação da localização do NF na interface. ${ }^{[4,13]}$. Este fenômeno ocorre devido à coalescência das fases. As fases aumentam de tamanho resultando em uma diminuição de tamanho da interface. Se o negro de fumo
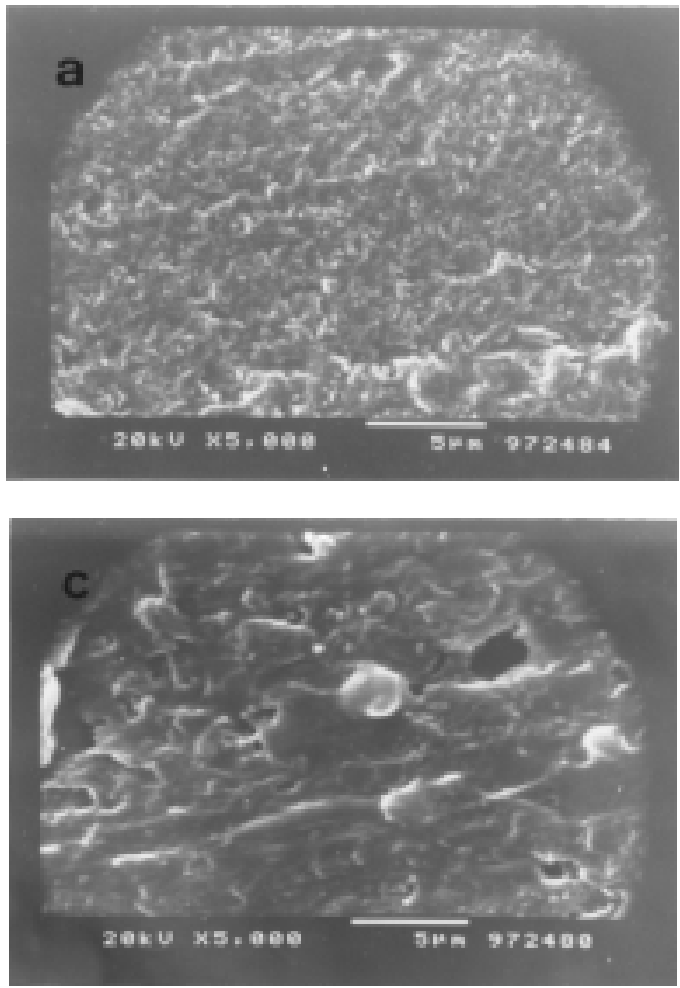

está situado na interface, a chance de contato entre as partículas aumenta consideravelmente com o decréscimo do espaço onde as partículas se concentram.

No caso das misturas PS/PBD moldadas tanto a $200^{\circ} \mathrm{C}$ como $250^{\circ} \mathrm{C}$ (Fig. $1 \mathrm{c}$ e $1 \mathrm{~d}$, respectivamente), a distribuição das fases é bem mais heterogênea, como é esperado para misturas heterogêneas. As partículas de negro de fumo não apresentaram distribuição uniforme e nem um caminho contínuo que pudesse resultar em condução elétrica, como no caso das misturas envolvendo PS/SB. Esta morfologia ajuda a explicar a baixa condutividade elétrica deste sistema, quando comparado ao PS/SB.

Para distinguir melhor a fase butadieno, as superfícies polidas das misturas PS/PBD foram submetidas a tratamento com hexano, a fim de extrair seletivamente a fase PBD. A Figura 2 apresenta as micrografias correspondentes, onde se observa com maior clareza a distribuição não uniforme dos domínios de PBD. Observa-se ainda que as cavidades formadas após tratamento com o solvente contém certa porção de PBD não extraído, indicando alguma adesão entre as fases. Considerando que as fases PS e PBD são reconhecidamente imiscíveis e que não existe qualquer interação química entre elas, a dificuldade em se extrair toda a fase PBD deve estar ligada à presença do negro de fumo.
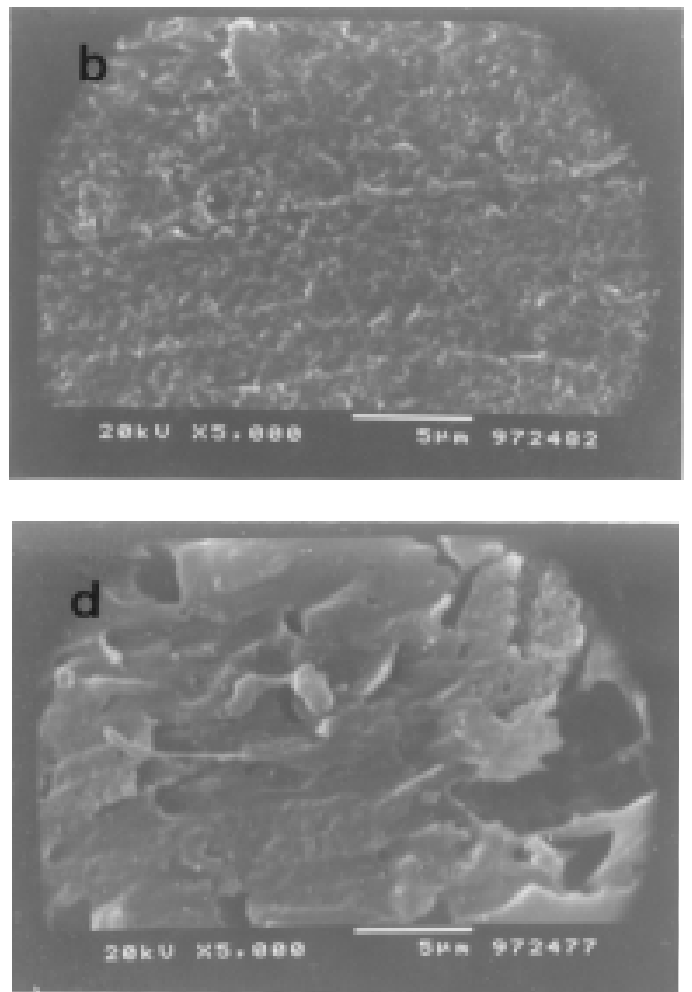

Figura 1. Microscopia eletrônica de varredura de misturas PS/SB (40:60\%) moldadas a $200^{\circ} \mathrm{C}$ (a) e $250^{\circ} \mathrm{C}$ (b) e de misturas $\mathrm{PS} / \mathrm{PBD}$ (90:10\%) moldadas a $200^{\circ} \mathrm{C}$ (c) e $250^{\circ} \mathrm{C}$ (d) 

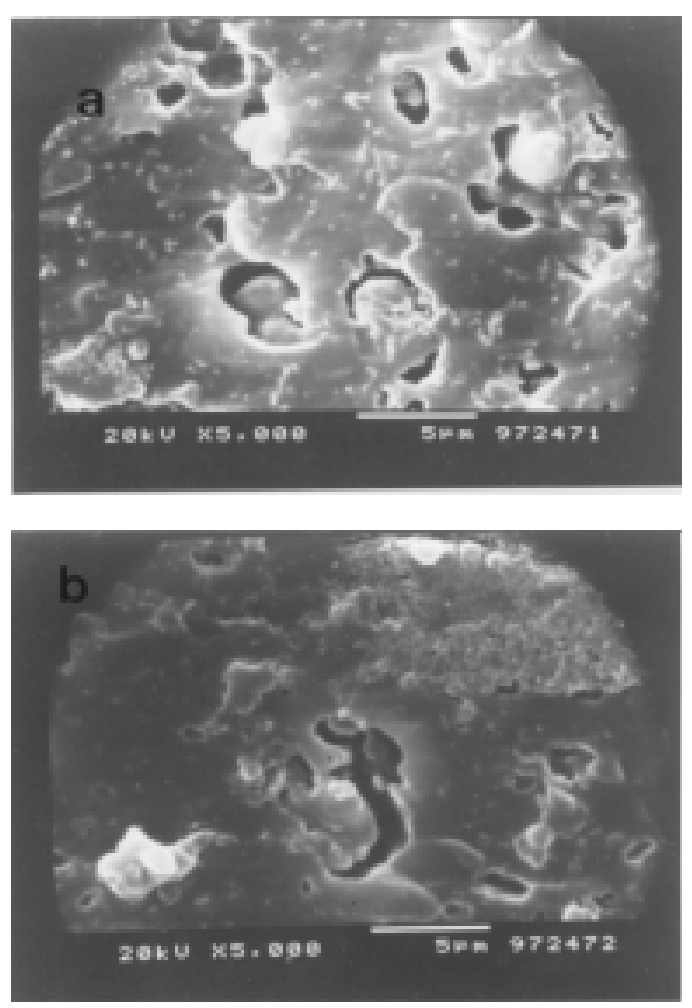

Figura 2. Microscopia eletrônica de varredura de fraturas de misturas PS/PBD, moldadas a $200^{\circ} \mathrm{C}$ (a) e $250^{\circ} \mathrm{C}$ (b), seletivamente extraídas com n-hexano.

Durante o processamento de elastômeros com negro de fumo, parte da borracha pode estar adsorvida à superfície do NF de tal forma que a sua separação através da extração com solvente torna-se muito difícil. Este fenômeno, conhecido como "bound rubber", é bastante discutido na literatura, e envolve interações essencialmente físicas entre polímero-negro de fumo, uma vez que estas são destruídas com o aumento da temperatura utilizada na solubilização. ${ }^{[22-25]}$ Para se determinar quantitativamente estas interações, costuma-se dissolver o material em um solvente adequado para o material polimérico. Após a solubilização e centrifugação, o polímero aderido à superfície do negro de fumo faz com que este se disperse na fase orgânica, permanecendo nesta forma mesmo após a centrifugação. A quantidade de NF constituinte desta fase dispersa é denominada $\mathrm{NF}_{\text {disp. }}$ À medida que aumenta a concentração de NF, aumenta o grau de interações polímero-NF, dando origem a uma massa completamente aderida e insolúvel, contendo polímero e negro de fumo. A formação de interações entre polímero-NF é favorecida quando NF possui grande área superficial e alta estrutura. ${ }^{[25]}$

Para avaliar a formação de "bound rubber", as misturas PS/SB e PS/PBD contendo $3 \%$ em peso de negro de fumo e moldadas a 200 e $250^{\circ} \mathrm{C}$, foram submetidas à extração com tolueno à temperatura ambiente durante $48 \mathrm{~h}$ e posteriormente centrifugadas. A camada orgânica foi separada e o processo repetido por mais três vezes. O resíduo obtido após a centrifugação foi seco, pesado e submetido à análise termogravimétrica (TGA). A Tabela 2 apresenta os dados quantitativos destes experimentos. Misturas constituídas de PS/SB apresentaram, após o fracionamento, uma parte do negro de fumo sob a forma de suspensão estável, que não conseguiu se separar mesmo após $2 \mathrm{~h}$ de centrifugação. A análise de TGA do material insolúvel indicou ainda a presença de polímero completamente aderido às partículas de negro de fumo $\left(R_{b}\right)$. Estes resultados sugerem uma forte interação entre polímero-NF. Aumentando a temperatura de moldagem, aumenta ainda mais a chance de interação, aumentando com isto o teor de $\mathrm{R}_{\mathrm{b}}$.

No caso de misturas envolvendo PS/PBD, não se observou a presença de partículas de NF suspensas na camada orgânica. Após a centrifugação, a camada de tolueno se apresentou completamente límpida. Entretanto, a quantidade de material polimérico insolúvel foi bem maior do que no caso anterior, sendo também superior à proporção de PBD na mistura. Estes resultados sugerem que parte do poliestireno na mistura se encontra também fortemente aderido ao sistema contendo negro de fumo e polibutadieno. Quando as mesmas amostras trituradas de PS/PBD foram submetidas à extração com MEK, toda a fase PS foi extraída, indicando que a interação entre PS e os demais componentes da mistura é de natureza puramente física, sendo destruída em solvente mais polar.

Os resultados experimentais apresentados anteriormente permitem concluir ainda que as interações entre os segmentos de polibutadieno e NF são bem mais fortes quando este polímero está na forma de homopolímero. Esta forte adesão pode explicar os maiores valores de limiar de percolação encontrados para este sistema. As partículas de NF se encontram bastante dispersas na fase PBD e circundadas por uma camada de polímero, dificultando assim a formação de uma rede de condução elétrica. Esta adesão foi

Tabela 2. Dados experimentais sobre as interações entre polímero-negro de fumo em função da temperatura de moldagem para misturas PS/SB e PS/PBD contendo $3 \%$ em peso de negro de fumo

\begin{tabular}{lcccc}
\hline \multicolumn{1}{c}{ Mistura } & \multicolumn{1}{c}{ PS/SB $(40: 60 \%)$} & PS/PBD (90:10\%) \\
\hline $\begin{array}{l}\text { Temperatura de } \\
\text { moldagem, }{ }^{\circ} \mathrm{C}\end{array}$ & 200 & 250 & 200 & 250 \\
Resistividade, $\Omega . c m$ & $1 \times 10^{13}$ & $1 \times 10^{7}$ & $1 \times 10^{13}$ & $1 \times 10^{13}$ \\
$\mathrm{R}_{\mathrm{b}}, \%$ & 2,00 & 3,20 & 23,0 & 22,4 \\
$\mathrm{NF}_{\text {gel }}, \%$ & 1,66 & 1,93 & 3,00 & 3,00 \\
$\mathrm{NF}_{\text {disp }}, \%$ & 1,34 & 1,07 & 0,00 & 0,00 \\
\hline
\end{tabular}


também sugerida anteriormente através das análises de microscopia eletrônica de varredura.

No caso da mistura PS/SB, é também constatada a adesão entre os segmentos de polibutadieno e as partículas de NF. Todavia, o menor peso molecular dos segmentos de PBD no copolímero e a proximidade dos segmentos de poliestireno, ligados quimicamente ao elastômero provavelmente dificultam a molhabilidade das partículas pela fase elastomérica. O contato entre as partículas de NF é facilitado e conseqüentemente, a rede de condução elétrica é formada a uma concentração bem menor de NF.

\section{Conclusões}

Como conclusões finais podemos afirmar que a concentração crítica de negro de fumo necessária para que ocorra a transição isolante-condutor (conhecida como limiar de percolação) é menor no caso da mistura envolvendo poliestireno e copolímero em bloco de estireno - butadieno. Misturas físicas constituídas de PS/PBD apresentam valores mais elevados de limiares de percolação devido provavelmente à maior dispersão do NF nos domínios de PBD e à forte interação física existente entre este homopolímero e as partículas de negro de fumo. Estas interações resultam na formação de uma superfície de polímero em torno das partículas condutoras e são provavelmente favorecidas pelo alto peso molecular do homopolímero de butadieno utilizado. As características morfológicas deste sistema dificultam a condução elétrica devido à não formação de uma rede contínua entre as partículas de negro de fumo.

\section{Agradecimentos}

Os autores agradecem ao CNPq, CAPES, CEPGUFRJ e PADCT-FINEP pelo apoio financeiro ao trabalho. Além disto, gostaríamos de agradecer à FAPERJ pela bolsa de iniciação científica fornecida ao aluno A. J. B. Ferreira.

\section{Referências Bibliográficas}

1. Probst, N.; Smet, H., Intern. Polym. Sci. Technol., 24, T/17 (1997)

2. Lee, B. L.; Polym. Eng. Sci., 32, 36 (1992)

3. Sumita, M.; Abe, H.; Kayaki, H.; Miyasaka, K.; J. Macromol. Sci.-Phys., B25, 171 (1986)
4. Gubbels, F.; Blacher, S.; Vanlathem, E.; Jérôme, R.; Deltour, R.; Brouers, F.; Teyssié, Ph. Macromolecules 28, 1559 (1995)

5. Thompson, C. M.; Allen, J. S.; - Rubber Chem. Technol., 67, 107 (1994)

6. Ircar, A. K.; Rubber Chem. Technol. 54, 820 (1981)

7. Medalia, A. I.; - Rubber Chem. Technol. 59: 432 (1986)

8. Myiasaka, K.; Watanabe, K.; Jojima, E.; Aida, H.; Sumita, M.; Ishikawa, K.; J. Mat. Sci., 17, 1610 (1982).

9. Kozlowski, M.; Kozlowska, A.; Macromol. Symp., 108, 261 (1996)

10. Lipatov, S.; Mamunya, Y.P.; Gladyreva, N.A.; Lebedev, V.; Polymer Sci. USSR, 25, 1714 (1983)

11. Geuskens, G.; Kezel, E.; Blacher, S.; Brouers, F.; Eur. Polym. J., 27, 1261(1991)

12. Sumita, M.; Sakata, K.; Asai, S.; Miyasaka, K.; Nakagawa, H.; Polym Bull 25:265 (1991)

13. Soares, B. G.; Gubbels, F.; Jérôme, R.; Teyssié, Ph.; Vanlathem, E.; Deltour, R.; Polym. Bull. 35, 223 (1995)

14. Soares, B. G.; Gubbels, F.; Jérôme, R.; Teyssié, Ph.; Vanlathem, E.; Deltour, R.; Rubber Chem. Technol. 70, 60 (1997)

15. Gamboa, K. M. N.; Ferreira, A. J. B.; Camargo Jr., S. S.; Soares, B. G.; Polym. Bull. 38, 95 (1997)

16. Soares, B. G.; Gamboa, K. M. N.; Ferreira, A. J. B.; Ueti, E.; Camargo Jr., S.; J. Appl. Polym. Sci., em publicação (1998)

17. Karásek, L.; Meissner, B.; Asai, S.; Sumita, M.; Polym. J., 28, 121 (1996)

18. Wang, M.-J.; Wolff, S.; Donnet, J.-B.; Rubber Chem. Technol 64, 714 (1991)

19. Dutta, N.K.; Choudhury, N.R.; Haidar, B.; Vidal, A.; Donnet, J.-B.; Delmotte, L.; Chezeau, J.M.; Polymer 35, 4293 (1994)

20. White, J. L.; Min, K.; Makromol. Chem., Macromol. Symp. - 16, 19 (1988)

21. Jorgensen, J. I.; Utracki, L. A.; Makromol. Chem., Macromol. Symp., 48/49, 189 (1991)

22. Stickney, P. B.; Falb, R. D.; Rubber Chem. Technol. 37, 1299 (1964)

23. Blow, C. M.; Polymer 14, 309 (1973)

24. Dannenberg, E. M.; Rubber Chem. Technol. 59, 512 (1986)

25. Wolff, S.; Wang, M.-J.; Tan, E.-H.; Rubber Chem. Technol. 66, 163 (1993) 